\title{
RETOS Y OPORTUNIDADES DE LA IMPLANTACIÓN DE LOS BANCOS DE AGUA EN ESPAÑA
}

\author{
Montilla-López, N.M. (1) (P), Gómez-Limón, J.A. (2), Gutiérrez-Martín, C. (3)
}

1 Investigador en formación, Universidad de Córdoba, Departamento de Economía, Sociología y Política Agrarias, Campus Universitario Rabanales, Ctra. N-IV Km 396, 14014 Córdoba, g02molon@uco.es

2 Catedrático de Universidad, Universidad de Córdoba, Departamento de Economía, Sociología y Política Agrarias, iglimon@uco.es

3 Profesor Ayudante Doctor, Universidad de Córdoba, Departamento de Economía, Sociología y Política Agrarias, es1gumac@uco.es

\section{Resumen}

Con el fin de conseguir una buena gobernanza del agua, que trate de alcanzar un equilibrio entre los objetivos de eficiencia económica y sostenibilidad ambiental, durante las últimas décadas se ha potenciado el uso de instrumentos económicos para una mejor gestión de la demanda de los recursos hídricos, dada la dificultad de seguir aumentando la oferta de los mismos. En este contexto, este trabajo se centra en los bancos de agua como mecanismo de mercado que aporta la necesaria flexibilidad institucional en la asignación de los recursos hídricos entre los diferentes usuarios. La investigación realizada en este sentido ha permitido desarrollar una tipología de los bancos de agua que actualmente operan a nivel mundial en base a sus características operativas. Esta caracterización ha permitido igualmente realizar un análisis crítico de las experiencias de bancos de agua implementadas hasta la fecha, con el fin poder valorar el desempeño de este instrumento económico para la mejora de la gestión pública del agua. La investigación llevada a cabo revela que los bancos de agua son un instrumento útil para la gestión de los recursos hídricos, aportando sugerencias para la mejora de su implementación operativa en España mediante la correspondiente reforma de la Ley de Aguas.

\section{1- Introducción y objetivos}

La escasez e incertidumbre de los recursos hídricos se han convertido en uno de los principales problemas al que debe enfrentarse la agricultura mundial en la actualidad. El aumento de la población y la consecuente demanda de alimentos ha provocado que a lo largo del siglo XX la superficie de regadío se incremente considerablemente a nivel global, convirtiéndose en la actividad humana con mayores extracciones y consumo de agua a nivel mundial (FAO 2012). Esta presión sobre los recursos hídricos es especialmente intensa en zonas semiáridas como California, Australia o España, donde la agricultura de regadío resulta especialmente competitiva, en las cuales los usos agrícolas representan hasta el $80 \%$ de los usos del agua. Esta situación de intensa demanda de recursos se ha denominado como "fase de madurez de la economía del agua" (Randall 1981), que puede llegar a provocar el "cierre" de las cuencas hidrográficas (imposibilidad de atender nuevas demandas sino es a costa de reducir la demanda de otros usuarios (Berbel et al. 2013). En 
este contexto resulta evidente la necesidad de promover una gestión eficiente del recurso, permitiendo su asignación preferente hacia los usos que generan mayor valor social, proceso en el que la agricultura de riego tendrá un papel central como mayor usuario del recurso en la actualidad.

En este sentido, la implementación de instrumentos políticos de demanda de agua, como la tarifación, la modernización de regadíos o los mercados de agua, se consideran prioritarios para la mejora del bienestar asociado al uso del recurso, dotando a las administraciones responsables de su gestión mecanismos para flexibilizar los sistemas de asignación de agua entre los usuarios. Dentro de este contexto, este trabajo se centra en el análisis de los bancos de agua, como un tipo específico de mercado de agua, que ya ha sido implementado con éxito en varios países al objeto de mejorar la gestión pública del recurso. Este instrumento se caracteriza por facilitar los cambios de uso del agua entre los diferentes usuarios, a través de un mecanismo controlado por la Administración pública competente en materia de agua (o de algún otro organismo), que actúa como gestora necesaria en todas las transacciones.

El presente trabajo se centra en estudiar la potencialidad de los bancos de agua (denominados centros de intercambio en la legislación española), como instrumento económico de gestión de la demanda enfocada hacia la satisfacción de las necesidades de agua en un contexto de cuencas cerradas (imposibilidad de aumento de la oferta de agua) y fuerte incertidumbre en cuanto la disponibilidad del recurso (impacto creciente de las sequias como consecuencia del cambio climático). Con este propósito se realiza un análisis crítico de la implementación real de los bancos de agua a nivel nacional e internacional, al objeto de poder analizar las ventajas e inconvenientes de este instrumento económico para la gestión de la escasez e incertidumbre de agua dentro de la política hidráulica. De esta manera se pretende concluir aportando una serie de sugerencias para la mejora del diseño de este instrumento económico dentro de la política del agua públicas en España, orientadas a la implementación operativa de los bancos de agua en nuestro país.

\section{2- Bancos de agua: concepto y tipologías}

La Teoría Económica demuestra que los mercados suponen un mecanismo eficiente de asignación de los recursos escasos (Coase 1960), siempre y cuando estos reúnan una serie de condiciones (mercados competitivos, que no produzcan externalidades y en ausencia de costes de transacción). Por este motivo los mercados de agua han sido señalados como un instrumento económico que puede mejorar la gestión del agua en contexto de escasez e incertidumbre sobre la disponibilidad del recurso.

Al hablar de mercados de agua, se hace en realidad referencia a todo un conjunto de instituciones que posibilitan intercambios de agua entre los usuarios. No obstante, de forma resumida los mercados de agua pueden dividirse en dos grandes categorías (Hadjigeorgalis 2009):

- Mercados descentralizados, en los que las transacciones se negocian bilateralmente entre los potenciales compradores y vendedores, bien para intercambiar cantidades de agua (cesión temporal de derechos de uso en mercados temporales o spot), o la propia titularidad del derecho de uso del recurso de manera definitiva (mercados permanentes o de derechos).

- Mercados centralizados o Bancos de agua, cuando la Administración (u otro agente) actúa como intermediaria necesaria en todas las operaciones de compraventa (Delacámara et al. 2015; MacDonnell 1995).

Los bancos de agua son pues un tipo de mercado de agua, que pueden implementarse tanto como único instrumento de mercado, como complemento a otros instrumentos económicos que promueven la gestión de la demanda de agua (McCrea \& Niemi 2007). 
Este trabajo se centra en el estudio de los bancos de agua ("water banks" en inglés), conocidos en España igualmente como centros de intercambio. Sin embargo, no conviene confundir este tipo de mercado de agua con el instrumento del "water banking", denominación anglosajona para describir una estrategia de gestión del recurso basada en el almacenamiento del recurso (Dellapenna 2000). Efectivamente, el water banking implica depositar derechos de agua, sea en papel o en volumen efectivo, en un "banco" (entendido éste como las reservas de agua en un embalse, acuífero, etc.), depósito que permite a su titular una amplia variedad de operaciones, entre las que se incluye el uso diferido del recurso y su transferencia a otros depositarios (Clifford et al. 2004).

Bajo el concepto general descrito anteriormente de "bancos de agua", en la práctica se engloban instituciones muy variadas. En este sentido, a continuación se analiza la heterogeneidad de este tipo de mercados de agua sobre la base de las experiencias que se han llevado a cabo hasta la fecha, analizando las principales características que los definen. Este análisis de las variables características de los bancos de agua ha permitido establecer distintas tipologías, tal y como se expone seguidamente.

En primer lugar debe señalarse que los bancos de agua se diferencian en función del tipo de iniciativa que hace posible su creación. En este sentido cabe considerar:

- Bancos de agua públicos, que son organizados y gestionados por una administración pública, normalmente aquella competente en materia de agua.

- Bancos de agua privados, que son organizados y gestionados por la iniciativa privada, principalmente mediante la actuación de organizaciones sin ánimo de lucro, tal y como las ONG dedicadas a la conservación del medioambiente.

Una segunda variable definitoria de los bancos de agua es el bien o derecho objeto de intercambio. Así pueden diferenciarse entre:

- Bancos de agua permanentes. Los vendedores transfieren al banco de agua la titularidad del derecho de uso o propiedad del recurso de manera definitiva. Los derechos así adquiridos por el banco pueden posteriormente asignarse, en su totalidad o parcialmente, a nuevos usuarios, bien mediante adquisición, bien mediante un sistema de concesión pública gratuita. Estos bancos están orientados a resolver problemas asociados a la escasez estructural del agua, tanto los de carácter económico como los ambientales (Bjornlund 2003; Wheeler et al. 2012), tal y como se comenta más adelante.

- Bancos de agua temporales o spot. Estos bancos se comportan de la misma manera que los bancos de agua permanentes pero, a diferencia de los anteriores, el objeto de las transferencias son cesiones temporales de los derechos de uso del agua (normalmente por una campaña de riego) o cantidades de agua concretas (spot). En ambos casos, su actividad se concentra en períodos de sequía, orientándose a mitigar los efectos de la escasez coyuntural del recurso.

- Bancos de contratos de opción. El banco de agua intercambia contratos que permiten a sus compradores tener la opción (pero no la obligación) de comprar agua al vendedor del contrato (titular de los derechos de uso del recurso), a cambio de un precio determinado o "prima" (Cui \& Schreider 2009; Israel \& Lund 1995; Jercich 1997). Si finalmente se ejecuta la mencionada opción de compra, el comprador pagará una compensación adicional al vendedor, denominado "precio de ejecución" o strike. Estos contratos permiten al comprador protegerse del riesgo de no disponer de agua suficiente para su actividad, permitiendo al mismo tiempo que el vendedor no pierda el derecho de propiedad o uso del agua.

Los bancos de agua pueden clasificarse igualmente según su finalidad. En este sentido cabe distinguir entre:

- Bancos de agua para la reasignación del recurso para usos productivos. Los intercambios de derechos que permiten los bancos de agua posibilitan la 
reasignación del recurso (temporal o permanentemente) en función de la oferta y la demanda de los usuarios (actuales y potenciales), favoreciendo la transferencia de agua desde usos de menor valor a otros de mayor valor. Estas trasferencias, en ausencia de externalidades negativas, permite la mejora de la eficiencia económica (mejora del valor de la producción) asociadas al uso de agua (Booker et al. 2005; Grafton et al. 2012; Wheeler et al. 2014).

- Bancos de agua para la consecución de fines ambientales. La operatividad de estos bancos se basa en la compra de derechos sin posterior reasignación de los mismos (Burke et al. 2004; Wheeler et al. 2013). De esta manera, este tipo de bancos permite solucionar problemas ambientales que puedan derivarse de la escasez de agua, tanto estructural (por la sobreasignación de recursos en la cuenca, mediante la compra de derechos permanentes) como coyuntural (caudales de estiaje insuficientes, mediante la compra de derechos temporales).

- Bancos de agua para gestión del riesgo de disponibilidad de agua. La incertidumbre climática e hidrológica intrínseca a la gestión de agua motiva la variabilidad interanual de la dotación del recurso, situación que les genera una importante exposición al riesgo a los usuarios, que justifica que estos tomen decisiones no eficientes desde una perspectiva económica (Alcón et al. 2014). Al objeto de minimizar decisiones subóptimas y mejorar la eficiencia en el uso del agua, este tipo de bancos se implementan mediante la negociación de contratos de opción sobre el agua. Con ello, este tipo de bancos de agua permite mejorar la garantía de suministro de los compradores de los contratos de opción (a base de empeorar la garantía de los vendedores de tales contratos), posibilitando de esta manera la transferencia efectiva del riesgo entre usuarios con distinto nivel de aversión al mismo (Howitt 1998; Ranjan 2010; Rey et al. 2016).

entre:

Finalmente, en función de la estrategia de gestión del mercado, cabe diferenciar

- Bancos de agua activos, aquellos donde los gestores del banco adoptan una estrategia proactiva como "creadores de mercado" (market-maker), comprando derechos de agua con su propio presupuesto, para posteriormente venderlos entre los usuarios potencialmente interesados. En este sentido, la entidad gestora del banco de agua persigue alcanzar una situación próxima al equilibrio de mercado que ocurrirá en un mercado descentralizado, tratando que el conjunto de operaciones de compra y venta no le suponga un coste neto (importe de la cifra de compras equivalente al importe de la cifra de ventas), o que tal coste no supere un presupuesto máximo dispuesto para tal fin. Debe señalarse que en estos casos el administrador del banco es el que establece las condiciones para la compra y venta de derechos (u opciones), de manera que estos bancos adquieren una característica de mercado monopolista, con un sistema de contratación de sentido único (Loomis et al. 2003). Así, en primer lugar, el banco actúa como único comprador de derechos u opciones de agua (monopsonio mediante ofertas públicas de compra), para posteriormente ser el único vendedor de tales derechos u opciones (monopolio mediante ofertas públicas de venta).

El sistema de compra puede variar en función de las características de la oferta pública: i) estableciendo el importe máximo de las compras (presupuesto), ya sea mediante un precio fijado o mediante un procedimiento de subasta (incrementos sucesivos del precio de adquisición hasta agotar el presupuesto asignado); ii) estableciendo el volumen máximo de agua que se desea adquirir, también mediante procedimientos de precio fijado o subasta; y iii) estableciendo un precio de mercado fijo de adquisición, sin limitaciones presupuestarias o de volúmenes de agua a adquirir. Las ofertas públicas también pueden diferenciarse por su carácter abierto o restringido; mientras en las primeras pueden acudir de manera voluntaria todos los titulares de derechos del ámbito territorial afectado por el banco (p. ej., una 
demarcación hidrográfica o usuarios de una acuífero), en las segundas sólo pueden hacerlo los usuarios autorizados para ello (p. ej., determinados tipos de usuarios). De manera análoga, las ofertas públicas de venta pueden diferenciarse en función tanto de las condiciones de precio y cantidad a contratar, como del carácter abierto/restringido de las mismas.

Este tipo de bancos de agua resultan útiles para incrementar la actividad de mercado (mejora de eficiencia económica) y ejercer un control más efectivo sobre las operaciones de mercado (minimización de externalidades y evitar la especulación).

- Bancos de agua pasivos, aquellos que no intervienen de forma activa en el mercado, limitando sus funciones a facilitar el contacto entre compradores y vendedores para la realización de operaciones en función de la demanda y la oferta existente en cada momento. En estos casos el papel del gestor del banco es únicamente como intermediario necesario de las compraventas (broker) y cámara de compensación (clearinghouse). En este contexto, las ofertas de compra y venta de derechos están basadas en un sistema de contratación de doble sentido, donde el banco ofrece la información de forma transparente (posiciones u ofertas de compra y de venta) para realizar las transacciones de forma similar como se hace en los mercados bursátiles. Así pues, mediante el cruce de las ofertas de compra y venta existentes, se establece un precio de mercado, al cual se intercambian todos los derechos de todos los agentes interesados en aceptar/pagar el precio de equilibrio alcanzado en cada momento (Bjornlund 2003).

Tras analizar las alternativas de diseño de los bancos de agua, y establecida la tipología sobre la heterogeneidad de características existentes, debe señalarse que no todas las combinaciones de variables definitorias de estos mercados se dan en la realidad. Efectivamente, de las experiencias implementadas hasta la fecha se evidencia que existe una fuerte correlación entre las variables arriba analizadas. Así por ejemplo, se puede comprobar como aquellos bancos creados para la resolución de problemas ambientales derivados de la sobreasignación de derechos son normalmente de iniciativa pública, donde se intercambian derechos de forma permanente y con una forma de gestión activa. Alternativamente, cuando se trata de minimizar las consecuencias económicas derivada de la de escasez coyuntural motivada por la sequía, los bancos de agua implementados son igualmente públicos, pero intercambiando derechos de agua temporales, bien a través de estrategias de gestión activa o pasiva.

\section{3- Los bancos de agua como instrumento para la gestión de la escasez}

Los períodos de sequías están siendo cada vez más comunes en todo el planeta como consecuencia del cambio climático. Hasta la fecha las estrategias de gestión de la escasez coyuntural han pasado por soluciones eminentemente estructurales, basadas medidas de oferta como el incremento de la capacidad de embalse, la explotación de aguas subterráneas y la reutilización de aguas residuales tratadas (Calatrava et al. 2011; Hanak et al. 2011). No obstante, evidencias recientes (Bjornlund 2003; Brewer et al. 2008; Harou et al. 2010; Martínez-Granados \& Calatrava 2014) demuestran que tales alternativas de gestión resultan mucho más costosas que las basadas en la gestión de la demanda, como son los mercados de agua. En este sentido cabe señalar los ejemplos ofrecidos por las experiencias internacionales basadas en bancos de agua, especialmente las de California y Australia, donde se ha puesto de manifiesto en éxito de este instrumento económico para la mejora de la gestión de sequías. De manera similar, también debe señalarse la interesante experiencia española en la implementación de bancos de agua, aunque en este caso la actividad ha sido muy escasa y los resultados controvertidos. Por este motivo el análisis crítico de la experiencia internacional resulta de especial interés para España, dadas las características climáticas (climas mediterráneos con alta variabilidad en la disponibilidad del recurso), hidrológicas (alta demanda de agua y cierre de cuencas) y productivas (usos agrarios 
altamente rentables en competencias con usos urbanos e industriales) comunes, con el propósito de mejorar la implementación de este tipo de mercados en nuestro país.

El estudio de las experiencias de los bancos de agua a nivel internacional y nacional permite evidenciar cómo su operatividad plantea una serie de ventajas e inconvenientes, que deben tenerse en cuenta a la hora de diseñar adecuadamente este instrumento económico al objeto de mejorar la gestión de la escasez. En este sentido se ha realizado una extensa revisión de la literatura existente al respecto, con el propósito de catalogar convenientemente las principales ventajas e inconvenientes asociadas a los bancos de agua, tal y como se sintetiza a continuación.

\section{Ventajas}

Los bancos de agua, al tratarse de un tipo de mercado de agua, comparten las mismas ventajas que estos, principalmente las relacionadas con la mejora de eficiencia en el uso del recurso (Bauer 1997; Easter \& Huang 2014; Easter et al. 1998, 1999; Garrick et al. 2009; Grafton et al. 2011; Howe et al. 1986; Michelsen \& Young 1993; Rosegrant \& Binswanger 1994):

$\checkmark$ Producen un aumento de las rentas a todos los usuarios del agua. La participación de los usuarios en el mercado es siempre voluntaria, circunstancia que justifica que todas las operaciones resulten beneficiosas (mejora de renta) para ambas partes.

$\checkmark$ Mejoran la eficiencia asignativa del recurso, favoreciendo transferencias de agua desde actividades de menor productividad marginal del agua hacia aquellas actividades con mayor productividad marginal, maximizando con ello el valor de la producción total derivada del uso del recurso disponible, lo que se traduce normalmente en una mejora del bienestar social.

$\checkmark$ Revelan el verdadero coste de oportunidad o coste de escasez del recurso, haciendo visible el valor económico para la sociedad, favoreciendo con ello un uso más racional del mismo.

$\checkmark$ Permiten aumentar la garantía de suministro a los usuarios más adversos al riesgo, ya que gracias a la posibilidad de intercambio reducen los efectos de la incertidumbre hidrológica.

$\checkmark$ Racionalizan la construcción de nuevas obras de infraestructuras hidráulica para el aumento de la oferta de agua, pues los mercados constituyen una alternativa a la construcción de costosas obras hidráulicas (precio de mercado inferior al coste marginal de los nuevos recursos).

Además de las ventajas comunes al resto de mercados de agua, los bancos de agua presentan una serie de ventajas específicas (Bauer 2010; Calatrava et al. 2011; Clifford et al. 2004; Gómez-Ramos 2013; O’Donnell \& Colby 2010):

$\checkmark$ Reducen los costes de transacción en las operaciones de intercambio, ya que al centralizar las compras y ventas de derechos (u opciones), reducen los costes derivados de la búsqueda de agentes interesados en participar, así como los administrativos relacionados con formalización de las operaciones y la verificación del cumplimiento de lo acordado. Esta reducción de los costes de transacción favorece los intercambios, haciendo de los bancos un instrumento más adecuado para la mejora de eficiencia en el uso del agua.

$\checkmark$ Los bancos de agua destacan en la reasignación de los derechos de agua con fines ambientales. Esta no es una función exclusiva de los gobiernos, ya que en muchos estados norteamericanos existen organizaciones sin ánimo de lucro que ejercen control sobre las externalidades ambientales y sociales como el incremento de los flujos de agua de los ríos a través de compras de derechos de agua en masas de agua sobreexplotadas para la recuperación de los caudales.

$\checkmark$ Mayor transparencia, ya que se consigue que los precios de compra y/o venta sean públicos y accesibles a todos los usuarios. 
$\checkmark$ Los bancos de agua de iniciativa pública, al estar gestionados por la Administración, aportan mayor seguridad y garantía de disponibilidad de recursos hídricos que los mercados de agua.

$\checkmark$ La implementación temprana de los bancos de agua en los primeros estadios de la sequía concienciaría de manera más efectiva al conjunto de usuarios sobre la necesidad de realizar esfuerzos para disminuir la demanda con el fin de mitigar los efectos negativos de un eventual agravamiento de la sequía.

Tras las ventajas descritas, podemos concluir que los bancos de agua proporcionan la flexibilidad necesaria para la gestión de los recursos hídricos, favoreciendo la reasignación del agua en zonas donde los recursos disponibles ya están asignados y siguen surgiendo nuevos usuarios que reclaman agua, incluido el medio ambiente (WWF España 2005).

\section{Inconvenientes}

Los mercados de agua presentan una serie de inconvenientes, que por ende son comunes a los bancos de agua. A continuación se hace una relación de los principales inconvenientes que muestran estos instrumentos económicos (Bjornlund \& McKay 2002; BOE 2001; Delacámara et al. 2015; Dinar et al. 1997; Hearne \& Easter 1997; Johansson 2000; Qureshi et al. 2009; Rosegrant \& Binswanger 1994; Thobani 1997; Tsur 2000):

$\checkmark$ Activación de "derechos durmientes" o "derechos de papel". Con este nombre se conocen aquellos derechos de agua asignados a usuarios que realmente no los utilizan. Así, si el destino fuera la reasignación de derechos, se incrementaría el uso de agua total en el sistema, mientras que si el destino fuera el incremento de los caudales de las masas de agua, su compra no tendría efecto.

$\checkmark$ Compraventa del total de la concesión en lugar el agua realmente utilizada. Vender el volumen correspondiente al derecho del agua (concesión) resultaría en una situación similar a la anterior en la que se pondrían en circulación derechos de agua no utilizados.

$\checkmark$ Externalidades ambientales negativas al realizar la compraventa de la dotación (agua realmente utilizada) en vez del agua consumida, que derivaría en la pérdida de retornos de agua en la zona cedente, degradando el medio ambiente al afectar al mantenimiento de los caudales en las zonas de origen.

$\checkmark$ Disminución global de los retornos. Realizar transferencias desde zonas de baja eficiencia en el uso del agua a zonas de mayor eficiencia puede disminuir los retornos de agua al medio de forma global.

$\checkmark$ Otra externalidad negativa es el impacto a terceros por el empeoramiento socioeconómico en la zona de origen, debido a la pérdida de empleo por el abandono de la actividad y despoblamiento como consecuencia de la pérdida de renta asociada al empleo.

$\checkmark$ Elevados costes de transacción asociados al intercambio de derechos del agua, que provocan rigidez en el mercado y pérdida de capacidad de respuesta ante situaciones inciertas. Estos costes de transacción incluyen: i) costes derivados de poner en contacto a compradores y vendedores, ii) costes administrativos asociados al contrato que ha de realizarse, y iii) coste de las infraestructuras para realizar las transferencias físicas de agua. Aunque, como ya se ha mencionado, los bancos de agua disminuirían parte de estos costes de transacción.

$\checkmark \quad$ Imperfecciones en el mercado. El flujo unidireccional del agua de los ríos implica que en principio solo puedan realizarse las transferencias de recursos dentro de una misma cuenca, y de los usuarios situados en las partes más elevadas hacia otros situados en zonas más bajas. No obstante, si hay suficientes recursos en la cabecera de la cuenca, también se pueden transferir derechos aguas arriba. Para solventar estos obstáculos sería necesario realizar costosas infraestructuras (que incrementarían aún más los costes de transacción). 
$\checkmark$ Fraudes en el uso del agua tras la venta del recurso. Si no existe un control adecuado, podría resultar que el cedente siga utilizando agua tras la venta de los derechos.

Además de los inconvenientes descritos, hay que tener en cuenta las barreras culturales (el agua no es considerada un bien objeto de comercio), físicas (inexistencia de las infraestructuras adecuadas para realizar las transacciones) y legales, que producen que los mercados de agua movilicen un volumen escaso de agua, considerándose por tanto "mercados estrechos". Así pues, si la actividad es escasa, se genera falta de competencia y dispersión en los precios, que dejan de ser un buen indicador de la escasez del recurso (Tisdell 2011).

Cabe destacar que los bancos de agua poseen unas características específicas que hacen que, además de tener los inconvenientes descritos para los mercados de agua, sea compleja su creación. Así, la creación de un banco de agua requiere de una gestión administrativa compleja, que sea capaz de gestionar los contratos entre compradores y vendedores, así como asegurar que todos los agentes implicados pueden participar en el mercado con los volúmenes de agua realmente utilizados, minimizando las externalidades negativas.

\section{Experiencia internacional}

Entre los países con mayor volumen de agua intercambiado se encuentran Australia y Chile, que puede llegar a superar el $10 \%$ del total de los recursos consumidos (Hadjigeorgalis \& Lillywhite 2004), siendo muy superior a los volúmenes que se movilizan en España. En este sentido, en los estados del oeste de los Estados Unidos, las transferencias de agua son similares a las de las cuencas del sureste español y no llegan a superar el 5\% del consumo total en los períodos más secos (Briscoe 1997; Hanak \& Stryjewski 2012).

Las experiencias en bancos de agua tanto en Australia como en Estados Unidos se pueden encuadrar según la tipología que se ha establecido. En la Tabla 1 se recogen de forma sintética la clasificación según la tipología y los estados en los que se han desarrollado los bancos de agua.

Tabla 1. Bancos de agua en el Oeste de los Estados Unidos.

\begin{tabular}{|c|c|c|c|c|c|c|c|c|c|c|c|c|c|c|c|c|}
\hline & & 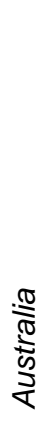 & 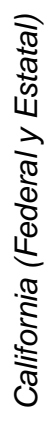 & 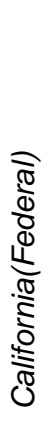 & 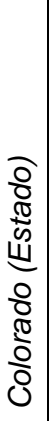 & 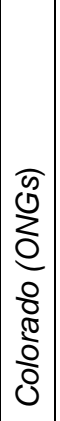 & 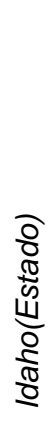 & 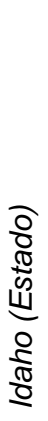 & 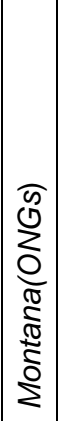 & 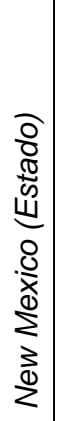 & 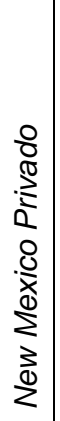 & 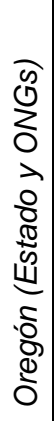 & 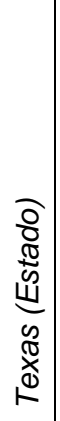 & 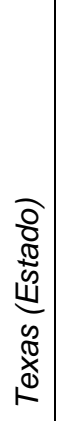 & 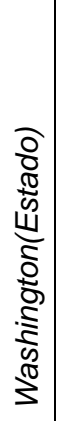 & 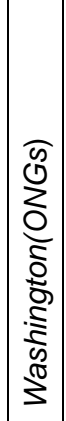 \\
\hline \multirow{3}{*}{$\begin{array}{l}\text { En función del bien } \\
\text { intercambiado }\end{array}$} & Permanentes & $x$ & $x$ & $x$ & & & & $x$ & & $x$ & & & $x$ & $x$ & & $x$ \\
\hline & Temporales o spot & $\mathrm{X}$ & $\mathrm{X}$ & $\mathrm{X}$ & $\mathrm{X}$ & $\mathrm{X}$ & $x$ & & $\mathrm{X}$ & $\mathrm{X}$ & $\mathrm{X}$ & $\mathrm{x}$ & $\mathrm{X}$ & & $\mathrm{X}$ & $\mathrm{X}$ \\
\hline & Opciones o futuros & & $\mathrm{X}$ & & & & & & & & & & & & $\mathrm{X}$ & \\
\hline \multirow{3}{*}{ Según la finalidad } & Reasignación del recurso & $\mathrm{X}$ & $\mathrm{X}$ & & $\mathrm{X}$ & & $\mathrm{X}$ & & & $\mathrm{X}$ & $\mathrm{X}$ & & $\mathrm{X}$ & & $\mathrm{X}$ & \\
\hline & Fin medioambiental & $\mathrm{X}$ & & $\mathrm{X}$ & & $\mathrm{X}$ & & $\mathrm{X}$ & $\mathrm{X}$ & $\mathrm{X}$ & & $\mathrm{x}$ & & $\mathrm{X}$ & & $\mathrm{X}$ \\
\hline & $\begin{array}{l}\text { Gestión del riesgo de } \\
\text { disponibilidad de agua }\end{array}$ & & $X$ & & & & & & & & & & & & $X$ & \\
\hline \multirow{2}{*}{ Operatividad del banco } & Activo & & $\mathrm{X}$ & $\mathrm{X}$ & & $\mathrm{X}$ & $\mathrm{X}$ & $\mathrm{X}$ & $\mathrm{X}$ & & & $\mathrm{x}$ & $\mathrm{X}$ & $\mathrm{X}$ & $\mathrm{X}$ & $\mathrm{X}$ \\
\hline & Pasivo & $\mathrm{X}$ & & & $\mathrm{X}$ & & & & & $\mathrm{X}$ & $\mathrm{X}$ & & & & & \\
\hline
\end{tabular}

Fuente: Elaboración propia. 


\section{4- Los bancos de agua en España}

La rigidez del sistema concesional español hace que sea necesario introducir flexibilidad, con el objetivo de permitir un uso más eficiente del agua que proporcione mayor satisfacción al conjunto de la sociedad. En este sentido, la Ley de Aguas 46/1999, de Reforma de la Ley de Aguas de 1985, rompe el principio de "la vinculación del agua a la tierra" y permite nuevas formas de reasignación de recursos hídricos mediante la regulación de mercados formales de agua ${ }^{1}$ a través de dos figuras diferentes: i) contratos de cesión de derechos de uso de agua (art. 67), que se corresponden con un diseño de mercado de derechos temporales o spot entre particulares y ii) los centros de intercambio (art. 71), que se ajustan a los bancos de agua objeto de este trabajo, donde pueden transferirse tanto derechos temporales como permanentes.

El Real Decreto-ley 15/2005, aprobó la constitución de los centros de intercambio de derechos de uso del agua como tipo de mercado de agua en situaciones excepcionales y urgentes para paliar los efectos de la sequía en determinadas cuencas (Segura, Júcar, Guadiana y Guadalquivir). Esta situación de excepcionalidad se fue prorrogando a través de sucesivos reales decreto-ley hasta noviembre 2008, cuando se consideró terminado el episodio de sequía. En el sistema original del TRLA no cabe reservar derechos para usos ambientales y es desde el Real Decreto Ley 9/2006, de 15 de septiembre, cuando los centros de intercambio ganan operatividad y se adoptan medidas urgentes para paliar los efectos producidos por la sequía en las poblaciones y en las explotaciones agrarias de regadío en determinadas cuencas hidrográficas. La última reforma de la Ley de Aguas española de mayo de 2012 se centra en los recursos de agua subterránea y pone de relieve la necesidad de simplificar y acelerar los procedimientos administrativos para añadir más flexibilidad y eficiencia al sistema de gestión del agua, incluyendo el fomento de la transformación de los derechos de aguas privadas en concesiones de agua pública (Rey et al. 2014).

En España se han desarrollado tres casos donde se han creado centros de intercambio en las cuencas de los ríos Guadiana, Júcar y Segura. Según la tipología establecida, se podrían clasificar estas tres experiencias como bancos de agua activos de iniciativa pública mediante ofertas públicas de adquisición de derechos (OPAD), cuya finalidad principal fue la mejora del medio ambiente. No obstante, hay algunas diferencias fundamentales que hacen que estos bancos hayan tenido características diferenciadas. La principal divergencia reside en que, a diferencia de los centros de intercambio del Júcar y Segura, donde se trataba de paliar un problema coyuntural de caudal en los ríos debido a la sequía, en el caso del Guadiana se trataba de un problema estructural de escasez por sobreexplotación del acuífero de la Mancha Occidental. Así, en el caso del Guadiana se estableció un centro de intercambio para la compra permanente de derechos de agua, cuya finalidad fue doble: la recuperación del acuífero y la reasignación de recursos, dotando de agua a algunos cultivos sociales. Por el contrario, en los casos del Júcar y Segura, al tratarse de un problema coyuntural, se realizaron adquisiciones temporales de derechos de agua. Además, cabe destacar que las adquisiciones de derechos en el Guadiana y Segura se realizaron mediante subastas, mientras que en el Júcar se realizaron a precio fijado en función de la distancia a la masa de agua afectada.

No obstante, se detectaron algunos inconvenientes en las OPAD del Guadiana, donde se compraron derechos que realmente no se utilizaban y además se detectó que, en algunos casos, el agua se continuó utilizando tras su venta. Sin embargo, en el caso del Júcar, se evitaron estos problemas gracias un mejor control de la elegibilidad de los cedentes y un mayor control sobre el uso posterior del agua.

\footnotetext{
${ }^{1}$ Los mercados formales son aquellos en los que la Administración pública interviene en su regulación (PalomoHierro et al. 2015).
} 
Además de la ley nacional, existe legislación específica que regula los bancos de agua en Andalucía en los distritos hídricos que dependen enteramente de la administración regional. En este contexto, la Ley 9/2010 de Aguas de Andalucía permite obviar la prelación de usos del agua, igualando en un mismo nivel de prioridad a los usos agrarios, industriales y de turismo, así como la obligatoriedad de ser concesionario para poder actuar como comprador, evitándose así asimetrías en el mercado. Además, permite adquirir agua de propiedad privada, transformándola en agua de propiedad pública.

A pesar de la posibilidad de creación de bancos de agua en España, la rigidez para su constitución ha impedido que se hayan desarrollado más experiencias, ya que es necesario que se den circunstancias de excepcionalidad para que el Ministro de Medio Ambiente proponga la creación de un banco de agua activo al Consejo de Ministros y haya un acuerdo formal para que sea constituido, además de la necesaria dotación presupuestaria.

\section{5- Lecciones aprendidas: directrices para la mejora de la normativa española}

A través del trabajo se han expuesto las ventajas y los inconvenientes de los bancos de agua según las experiencias tanto a nivel nacional como internacional. Antes de finalizar, en este apartado cabe comentar opciones de mejora en base a los inconvenientes encontrados en el funcionamiento de los bancos de agua:

1) Facilitar las gestiones para la constitución de un banco de agua.

2) Posibilitar que los organismos no gubernamentales puedan gestionar bancos de agua, tanto para fines medioambientales como para la reasignación del recurso.

3) Establecer de forma continua los bancos de agua, no solo en ocasiones excepcionales, con el objeto de atraer a los usuarios del agua a participar en el mercado con mayor regularidad y así poder evaluar si este instrumento resultaría realmente útil para la gestión del recurso en España.

4) Establecer un método ágil, apoyado en un sistema de información geográfica, para verificar con rapidez la información requerida para aprobar una adquisición de derechos por parte de un banco de agua (derechos de uso de agua, localización de las explotaciones agrarias, uso efectivo del derecho, infraestructura existente, etc.), evitando entre otros problemas la compraventa de derechos de agua no utilizados.

5) Eliminar barreras físicas, estableciendo la infraestructura necesaria y capacidad hidráulica para que las transferencias del recurso sean eficientes.

6) Transferir solo la fracción de agua consumida, y no la concesión o la dotación completa, evitando por una parte que se transfieran derechos no utilizados (al limitarlo a la dotación) y evitando que desde la zona de origen se transfiera la fracción de agua correspondiente a los flujos de retorno al medio (al limitarlo al agua realmente consumida), de manera que se minimicen o anulen los efectos ambientales en la zona de origen. En este sentido, la Ley de Aguas establece que solo se podrá ceder el derecho de uso correspondiente al agua utilizada en un periodo de referencia, pero habría que ir más allá y solo permitir la cesión de los volúmenes consumidos.

7) Establecer un método de control para evitar fraudes tras la venta del derecho de uso.

8) Incrementar la transparencia para disminuir los costes de transacción derivados de poner en contacto a compradores y vendedores. En este sentido, sería necesario definir claramente las zonas donde se puede comprar y/o vender derechos de agua a través del banco de agua con la infraestructura existente.

Para finalizar con la relación sobre las lecciones aprendidas, cabe destacar que resultaría necesaria una reforma de la Ley de Aguas que recoja todos los puntos anteriores, estableciendo leyes robustas para la mejora de la gobernanza que consiga la conexión entre instituciones, la eficiencia económica y la sostenibilidad ambiental. 


\section{6- Conclusiones}

El trabajo se ha centrado en los bancos de agua como mecanismo de mercado implementado ya en varios países al objeto de facilitar la reasignación del recurso entre los distintos usuarios. Así pues, la primera conclusión que se deriva del documento es que los bancos de agua han sido una herramienta útil a nivel internacional para resolver problemas de escasez de recursos hídricos, tanto coyuntural como estructural.

La introducción de este tipo de instrumentos mejora de la eficiencia asignativa del uso del agua, ya que permite que emerjan los costes de oportunidad del recurso, permitiendo su reasignación desde actividades de menor valor hacia usos de mayor valor donde el agua es pagada a precios más altos y su rendimiento es mayor. También permiten reducir conflictos sociales, poniendo en contacto a compradores y vendedores, evitando así la especulación de precios y cantidades que se intercambian, ya que es la Administración (u otro organismo) la que adquiere un papel central proporcionando agilidad y una rápida respuesta frente a situaciones excepcionales tales como la sequía.

En este contexto, aportan más flexibilidad a la gestión del agua, reduciendo la variabilidad en el beneficio, mejorando el bienestar social asociado al uso del agua y siendo además mejores que los mercados de derechos de agua a la hora de estabilizar la disponibilidad de agua de un usuario, ya que disminuyen los costes de transacción asociados a la puesta en contacto entre usuarios y los costes administrativos, aportando mayor transparencia.

La investigación llevada a cabo revela que al mercado español aún le queda un largo recorrido para conseguir alcanzar el buen funcionamiento de los bancos de agua, tal y como se ha alcanzado en otras zonas como California y Australia. El número de operaciones llevadas a cabo en España han sido escasas, y no es posible analizar y evaluar con exactitud si esta herramienta sería mejor que otras medidas. Sin embargo, creemos que un diseño adecuado de los bancos de agua, una adecuada regulación y una actuación activa por parte de la Administración (u otro organismo), pueden conseguir la eficiencia económica y la sostenibilidad ambiental, objetivos básicos de la gestión pública de los recursos hídricos.

Para ello, puede resultar interesante establecer, bajo un protocolo adecuado, un banco de agua de manera continua que promueva la participación activa de todas las partes implicadas. Así, podrían obtenerse de forma regular evaluaciones anuales y analizar con mayor detalle las ventajas e inconvenientes que se derivan de su uso, de las que se podrían extraer aportaciones que mejoren el diseño de este instrumento económico dentro de la política del agua.

\section{7- Agradecimientos}

Esta investigación ha sido financiada por el Ministerio de Economía y Competitividad (MINECO) y el Fondo Europeo de Desarrollo Regional (FEDER), a través del Proyecto de Investigación AGL2013-48080-C2-1-R y de la Ayuda Predoctoral FPI asociada al mismo.

\section{8- Bibliografía}

Alcón, F., Tapsuwan, S., Brouwer, R. \& de Miguel, M.D. (2014). Adoption of irrigation water policies to guarantee water supply: A choice experiment. Environmental Science and Policy, 44, 226-236.

Bauer, C.J. (1997). Bringing water markets down to earth: The political economy of water rights in Chile, 1976-1995. World Development, 25(5), 639-656.

Bauer, C.J. (2010). El abanico de bancos de aguas en las Américas. Estudios de economía aplicada, 28(2), 227-236.

Berbel, J., Pedraza, V. \& Giannoccaro, G. (2013). The trajectory towards basin closure of a European river: Guadalquivir. International Journal of River Basin Management, 11(1), 111-119. 
Bjornlund, H. (2003). Efficient water market mechanisms to cope with water scarcity. International Journal of Water Resources Development, 19(4), 553-567.

Bjornlund, H. \& McKay, J. (2002). Aspects of water markets for developing countries: Experiences from Australia, Chile, and the US. Environment and Development Economics, 7(4), 769-795.

BOE. (2001). Real Decreto Legislativo 1/2001, de 20 de julio, por el que se aprueba el texto refundido de la Ley de Aguas.: Boletín Oficial del Estado n 176, de 24/07/2001.

Booker, J.F., Michelsen, A.M. \& Ward, F.A. (2005). Economic impact of alternative policy responses to prolonged and severe drought in the Rio Grande Basin. Water Resources Research, 41(2), W02026.

Brewer, J., Glennon, R., Ker, A. \& Libecap, G.D. (2008). 2006 Presidential address. Water markets in the West: Prices, trading, and contractual forms. Economic Inquiry, 46(2), 91-112.

Briscoe, J. (1997). Managing water as an economic good: Rules for reformers. Water Supply, 15(4), 153-172.

Burke, S.M., Adams, R.M. \& Wallender, W.W. (2004). Water banks and environmental water demands: Case of the Klamath project. Water Resources Research, 40(9), W09S02.

Calatrava, J., Guillem, A. \& Martínez-Granados, D. (2011). Análisis de alternativas para la eliminación de la sobreexplotación de acuíferos en el Valle de Guadalentín. Economía Agraria y Recursos Naturales, 11(2), 33-62.

Clifford, P., Landry, C.J. \& Larsen-Hayden, A. (2004). Analysis of water banks in the western states. Olympia, USA: Washington State University, Department of Ecology.

Coase, R.H. (1960). The problem of social cost. Journal of Law and Economics, 3(1), 1-28.

Cui, J. \& Schreider, S. (2009). Modelling of pricing and market impacts for water options. Journal of Hydrology, 371(1-4), 31-41.

Delacámara, G., Gómez, C.M. \& Maestu, J. (2015). Water trading opportunities and challenges in Europe. In K. Burnett, R.E. Howitt, J.A. Roumasset, \& C.A. Wada (Eds.), Routledge handbook of water economics and institutions (pp. 281-295). Oxon, UK: Routledge.

Dellapenna, J.W. (2000). The importance of getting names right: The myth of markets for water. William \& Mary Environmental Law and Policy Review, 25(2), 317-377.

Dinar, A., Rosegrant, M.W. \& Meinzen-Dick, R. (1997). Water allocation mechanisms: Principles and examples. Washington D.C.: The World Bank.

Easter, K.W. \& Huang, Q. (2014). Water markets for the 21st century: What have we learned? New York: Springer.

Easter, K.W., Rosegrant, M.W. \& Dinar, A. (1998). Markets for water: Potential and performance. Boston: Kluwer Academic Publishers.

Easter, K.W., Rosegrant, M.W. \& Dinar, A. (1999). Formal and informal markets for water: Institutions, performance, and constraints. The World Bank Research Observer, 14(1), 99-116.

FAO. (2012). Irrigation water requirement and water withdrawal by country. Rome: FAO.

Garrick, D., Siebentritt, M.A., Aylward, B., Bauer, C.J. \& Purkey, A. (2009). Water markets and freshwater ecosystem services: Policy reform and implementation in the Columbia and Murray-Darling Basins. Ecological Economics, 69(2), 366-379.

Gómez-Ramos, A. (2013). Drought management uncertainty and option contracts. In J. Maestu (Ed.), Water trading and global water scarcity: International experiences (pp. 286-297). Oxon, UK: RFF Press.

Grafton, R.Q., Libecap, G.D., Edwards, E.C., O'Brien, R.J. \& Landry, C.J. (2012). Comparative assessment of water markets: Insights from the Murray-Darling Basin of Australia and the Western USA. Water Policy, 14(2), 175-193.

Grafton, R.Q., Libecap, G.D., McGlennon, S., Landry, C.J. \& O'Brien, R.J. (2011). An integrated assessment of water markets: A cross-country comparison. Review of Environmental Economics and Policy, 5(2), 219-239.

Hadjigeorgalis, E. (2009). A place for water markets: Performance and challenges. Review of Agricultural Economics, 31(1), 50-67. 
Hadjigeorgalis, E. \& Lillywhite, J. (2004). The impact of institutional constraints on the Limarí River Valley water market. Water Resources Research, 40(5), W05501.

Hanak, E., Lund, J.R., Thompson, B., Cutter, W.B., Gray, B., Houston, D., Howitt, R.E., Jessoe, K., Libecap, G.D., Medellín-Azuara, J., Olmstead, S.M., Sumner, D., Sunding, D., Thomas, B. \& Wilkinson, R. (2011). Managing California's water: From conflict to reconciliation. San Francisco, USA: Public Policy Instititute of California.

Hanak, E. \& Stryjewski, E. (2012). California's water market, by the numbers: Update 2012. San Francisco, USA: Public Policy Institute of California.

Harou, J.J., Medellín-Azuara, J., Zhu, T., Tanaka, S.K., Lund, J.R., Stine, S., Olivares, M.A. \& Jenkins, M.W. (2010). Economic consequences of optimized water management for a prolonged, severe drought in California. Water Resources Research, 46(5), W05522.

Hearne, R.R. \& Easter, K.W. (1997). The economic and financial gains from water markets in Chile. Agricultural Economics, 15(3), 187-199.

Howe, C.W., Schurmeier, D.R. \& Shaw, W.D. (1986). Innovate approaches to water allocation: The potenctial for water markets. Water Resources Research, 22(4), 439445.

Howitt, R.E. (1998). Spot prices, option prices, and water markets: an analysis of emerging markets in California. In K.W. Easter, M.W. Rosegrant, \& A. Dinar (Eds.), Markets for water-potential and performance (pp. 119-140). New York: Springer.

Israel, M. \& Lund, J.R. (1995). Private water leasing: Working within the prior appropriation system to restore stream flows. Natural Resources Journal, 35, 1-32.

Jercich, S.A. (1997). California's 1995 water bank program: Purchasing water supply options. Journal of Water Resources Planning and Management, 123(1), 59-65.

Johansson, R.C. (2000). Pricing irrigation water. A literature survey. Washington: The World Bank.

Loomis, J.B., Quattlebaum, K., Brown, T.C. \& Alexander, S.J. (2003). Expanding institutional arrangements for acquiring water for environmental purposes: Transactions evidence for the Western United States. International Journal of Water Resources Development, 19(1), 21-28.

MacDonnell, L.J. (1995). Water banks: Untangling the Gordian knot of Western water. In R.M.M.L. Foundation (Ed.), Proceedings of the Forty-First Annual Rocky Mountain Mineral Law Institute (pp. 1-63). Denver, Colorado: Mineral Law Institute.

Martínez-Granados, D. \& Calatrava, J. (2014). The role of desalinisation to address aquifer overdraft in SE Spain. Journal of Environmental Management, 144, 247-257.

McCrea, M.E. \& Niemi, E. (2007). Technical report on market-based reallocation of water resources alternative. A component of the Yakima River Basin storage feasibility study. Olympia, USA: Washington State University Department of Ecology.

Michelsen, A.M. \& Young, R.A. (1993). Optioning agricultural water rights for urban water supplies during drought. American Journal of Agricultural Economics, 75(4), 10101020.

O'Donnell, M. \& Colby, B. (2010). Water banks: A tool for enhancing water supply reliability. Tucson: University of Arizona, Department of Agricultural and Resource Economics.

Palomo-Hierro, S., Gómez-Limón, J.A. \& Riesgo, L. (2015). Water markets in Spain: Performance and challenges. Water, 7(2), 652-678.

Qureshi, M.E., Shi, T., Qureshi, S.E. \& Proctor, W. (2009). Removing barriers to facilitate efficient water markets in the Murray-Darling Basin of Australia. Agricultural Water Management, 96(11), 1641-1651.

Randall, A. (1981). Property entitlements and pricing policies for a maturing water economy. Australian Journal of Agricultural Economics, 25(3), 195-220.

Ranjan, R. (2010). Factors affecting participation in spot and options markets for water. Journal of Water Resources Planning and Management, 136(4), 454-462.

Rey, D., Calatrava, J. \& Garrido, A. (2016). Optimisation of water procurement decisions in an irrigation district: The role of option contracts. Australian Journal of Agricultural and Resource Economics, 59, 1-25. 
Rey, D., Garrido, A. \& Calatrava, J. (2014). The water markets in Spain: Moving towards 21st century mechanisms and approaches with 20th century regulations. In K.W. Easter \& $\mathrm{H}$. Qiuqiong (Eds.), Water markets for the 21st century: What have we learned? (pp. 127-147). New York: Springer.

Rosegrant, M.W. \& Binswanger, H.P. (1994). Markets in tradable water rights: Potential for efficiency gains in developing country water resource allocation. World Development, 22(11), 1613-1625.

Thobani, M. (1997). Formal water markets: Why, when, and how to introduce tradable water rights. The World Bank Research Observer, 12(2), 161-179.

Tisdell, J.G. (2011). Water markets in Australia: An experimental analysis of alternative market mechanisms. Australian Journal of Agricultural and Resource Economics, 55(4), 500-517.

Tsur, Y. (2000). Water regulation via pricing: The role of implementation costs and asymmetric information. In A. Dinar (Ed.), The Political Economy of Water Pricing Reforms (pp. 105-120). New York: Oxford University Press.

Wheeler, S.A., Garrick, D., Loch, A. \& Bjornlund, H. (2013). Evaluating water market products to acquire water for the environment in Australia. Land Use Policy, 30(1), 427436.

Wheeler, S.A., Loch, A., Zuo, A. \& Bjornlund, H. (2014). Reviewing the adoption and impact of water markets in the Murray-Darling Basin, Australia. Journal of Hydrology, 518, 2841.

Wheeler, S.A., Zuo, A., Bjornlund, H. \& Lane Miller, C. (2012). Selling the farm silver? Understanding water sales to the Australian Government. Environmental and Resource Economics, 52(1), 133-154.

WWF España. (2005). Los mercados de aguas y la conservación del medio ambiente: Oportunidades y retos para su implantación en España. Madrid: WWF España. 